\title{
Regular algebras of dimension 2, the generalized eigenvalue problem and Padé interpolation
}

Alexei ZHEDANOV

Donetsk Institute for Physics and Technology, Donetsk 83114, Ukraine

This article is a part of the special issue titled "Symmetries and Integrability of Difference Equations (SIDE VI)"

\begin{abstract}
We consider the generalized eigenvalue problem $A \psi=\lambda B \psi$ for two operators $A, B$. Self-similar closure of this problem under a simplest Darboux transformation gives rise to two possible types of regular algebras of dimension 2 with generators $A, B$. Realization of the operators $A, B$ by tri-diagonal operators leads to a theory of biorthogonal rational functions. We find the general solution of this problem in terms of the ordinary and basic hypergeometric functions. In special cases we obtain general Padé interpolation tables for the exponential and power function on uniform and exponential grids.
\end{abstract}

\section{Generalized eigenvalue problem and its Darboux trans- formations}

Let $A, B$ be two operators in some linear space $L$ (either finite- or infinite-dimensional) . The linear combination $Y(\lambda)=A-\lambda B$ is called a linear pencil [6]. Assume that a vector $\psi(\lambda)$ belongs to a kernel space of the operator $Y(\lambda)$. Clearly

$$
A \psi(\lambda)=\lambda B \psi(\lambda) .
$$

Thus the vector $\psi(\lambda)$ is a solution of the generalized eigenvalue problem (GEVP) (1.1) [2], [18]. When $B=I$, where $I$ is identical operator, then GEVP is reduced to the ordinary eigenvalue problem for then operator $A: A \psi(\lambda)=\lambda \psi(\lambda)$. The GEVP arises in many problems in the theory of separation of variables in PDE and in the mechanical vibrations $[2],[6]$.

Assume that a scalar product $\langle\psi, \chi\rangle$ is introduced on the space $L$. Then it is possible to consider the conjugated GEVP

$$
A^{*} \psi^{*}(\lambda)=\lambda B^{*} \psi^{*}(\lambda),
$$

for some vectors $\psi^{*}(\lambda)$, where $A^{*}, B^{*}$ are conjugated operators defined by $\left\langle A^{*} \psi, \chi\right\rangle=$ $\langle\psi, A \chi\rangle$ (note that we define the conjugated operator without taking complex conjugates, 
so, e.g. in the finite-dimensional case the conjugated matrix coincides with the transposed matrix). It is then elementary to verify the biorthogonality property

$$
\left\langle\psi(\lambda), B^{*} \psi^{*}(\mu)\right\rangle=0, \quad \text { if } \quad \mu \neq \lambda
$$

Equivalently, we can write

$$
\langle\psi(\lambda), \phi(\mu)\rangle=0, \quad \text { if } \quad \mu \neq \lambda
$$

where

$$
\phi(\mu) \equiv B^{*} \psi^{*}(\mu)
$$

We thus have two sets $\psi(\lambda)$ and $\phi(\lambda)$ of biorthogonal vectors with respect to scalar product $\langle\psi, \chi\rangle$.

The GEVP (1.1) possesses an important property of projective invariance. Indeed, define a new pair of operators

$$
C=\alpha A+\beta B, \quad D=\gamma A+\delta B,
$$

where $\alpha, \beta, \gamma, \delta$ are arbitrary complex parameters such that $\alpha \delta-\gamma \beta \neq 0$. Then the vector $\psi(\lambda)$ satisfies GEVP

$$
C \psi(\lambda)=\tilde{\lambda} D \psi(\lambda),
$$

where

$$
\tilde{\lambda}=\frac{\alpha \lambda+\beta}{\gamma \lambda+\delta} .
$$

This property allows one to choose arbitrary linear transformations of initial operators $A, B$ which leads merely to the Möbius transformation (1.8) of the spectral parameter $\lambda$ while the eigenvector $\psi(\lambda)$ remains the same.

Define the so-called Darboux transformations for GEVP (1.1) (for details see, e.g. [20]). Assume that four operators $T^{(1)}, T^{(2)}, \tilde{A}, \tilde{B}$ exist such

$$
\tilde{A} T^{(1)}=T^{(2)} A, \quad \tilde{B} T^{(1)}=T^{(2)} B
$$

Then it is elementary to verify that the vector

$$
\tilde{\psi}(\lambda) \equiv T^{(1)} \psi(\lambda)
$$

satisfies

$$
\tilde{A} \tilde{\psi}(\lambda)=\lambda \tilde{B} \tilde{\psi}(\lambda) .
$$

Thus the operator $T^{(1)}$ transforms a generalized eigenvector $\psi(\lambda)$ to a new generalized eigenvector $\tilde{\psi}(\lambda)$ of a new GEVP (1.11) with the same eigenvalue $\lambda$. Applying this procedure step-by-step, we can construct a set of GEVP

$$
A_{n} \psi_{n}(\lambda)=\lambda B_{n} \psi_{n}(\lambda), \quad n=0,1, \ldots
$$


such that

$$
A_{n+1} T_{n}^{(1)}=T_{n}^{(2)} A_{n}, \quad B_{n+1} T_{n}^{(1)}=T_{n}^{(2)} B_{n},
$$

where eigenvectors $\psi_{n}$ are related as

$$
\psi_{n+1}(\lambda)=T_{n}^{(1)} \psi_{n}(\lambda) .
$$

Thus the operator $T_{n}^{(1)}$ can be considered as the upward operator, i.e. $n \rightarrow n+1$ on the space of eigenvector $\psi_{n}$.

Note that the vector $T_{n}^{(2)^{*}} \psi_{n+1}^{*}(\lambda)$ belongs to a kernel space of the operator $Y_{n}^{*}(\lambda)=$ $A_{n}^{*}-\lambda B_{n}^{*}$. Thus the operator $T_{n}^{(2)^{*}}$ can be considered as "backward" operator, i.e. $n+1 \rightarrow$ $n$ (but acting on the space of conjugated eigenvectors $\psi_{n}^{*}$ ).

There is a special case of the Darboux transformations (1.9) when

$$
T_{1}=B, \quad T_{2}=\tilde{B}
$$

Then the second relation in (1.9) holds automatically and we need the only condition

$$
\tilde{A} B=\tilde{B} A .
$$

In this case

$$
\tilde{\psi}(\lambda)=B \psi(\lambda) .
$$

Repeating this procedure we obtain the chain of vectors $\psi_{n+1}(\lambda)=B_{n} \psi_{n}(\lambda), n=0,1, \ldots$ satisfying GEVP (1.12) with the condition

$$
A_{n+1} B_{n}=B_{n+1} A_{n}
$$

Consider a simple example of the Darboux transformation of the type (1.15). Let $A=\partial_{x}^{N}+\sum_{k=0}^{N-1} a_{k}(x) \partial_{x}^{k}$ be a differential operator of the order $N$ with unity coefficient at the highest derivative. Consider GEVP of the form

$$
A \psi(x ; \lambda)=\lambda b(x) \psi(x ; \lambda),
$$

where the operator $B$ coincides with multiplication by a function $b(x)$. Relation (1.16) reads

$$
\left(\partial_{x}^{N}+\sum_{k=0}^{N-1} \tilde{a}_{k}(x) \partial_{x}^{k}\right) b(x)=\tilde{b}(x)\left(\partial_{x}^{N}+\sum_{k=0}^{N-1} a_{k}(x) \partial_{x}^{k}\right),
$$

where $\tilde{a}_{k}(x), \tilde{b}(x)$ are some functions. Comparing terms in (1.20) at highest order $N$ we conclude that $\tilde{b}(x)=b(x)$. Hence in this case the transformed operator $\tilde{A}$ is merely a similarity transformation $b(x) A b^{-1}(x)$ of the differential operator $A$. Of course, in this simple case the result is obvious, but in other cases the Darboux transformations (1.9) can lead to nontrivial solutions of initial GEVP. 


\section{GEVP for tri-diagonal operators and biorthogonal ratio- nal functions}

In this section we consider the case when both $A$ and $B$ operators can be presented as tri-diagonal operators acting on some discrete basis $e_{n}, n=0,1, \ldots$ :

$$
A e_{n}=a_{n+1}^{(1)} e_{n+1}+b_{n}^{(1)} e_{n}+c_{n}^{(1)} e_{n-1}, \quad B e_{n}=a_{n+1}^{(2)} e_{n+1}+b_{n}^{(2)} e_{n}+c_{n}^{(2)} e_{n-1}
$$

with some complex coefficients $a_{n}^{(i)}, b_{n}^{(i)}, c_{n}^{(i)}, i=1,2$. Of course, it is assumed that $c_{0}^{(1)}=$ $c_{0}^{(2)}=0$ which means that the vector $e_{0}$ is the lowest in the basis $e_{n}$. The matrices $A, B$ may be either finite- or infinite-dimensional. In the first case we have additional conditions

$$
a_{N}^{(1)}=a_{N}^{(2)}=0
$$

for some $N=1,2, \ldots$, where $N$ is dimension of the matrices $A, B$. The corresponding basis in this case is constructed from $N$ linearly independent vectors $e_{0}, e_{1}, \ldots, e_{N-1}$. For the infinite-dimensional case we have $a_{n}^{(1)} a_{n}^{(2)} \neq 0$ for all $n=1,2, \ldots$.

To avoid triviality we should assume that operators $A, B$ cannot be decomposed as $A=L R_{1}, B=L R_{2}$, where $L, R_{1}, R_{2}$ are two-diagonal operators, because otherwise we will deal with rather trivial GEVP $R_{1} \psi=\lambda R_{2} \psi$ for two-diagonal operators.

In $[20]$ it was shown that GEVP for such tri-diagonal operators leads to a theory of biorthogonal rational functions. To see this we present the eigenvector $\psi(\lambda)$ as

$$
\psi(\lambda)=\sum_{k=0}^{N-1} R_{k}(\lambda) e_{k},
$$

where $R_{k}(\lambda)$ are expansion coefficients and $N$ may be either finite or infinite. Substituting (2.3) into (1.1) we arrive at the recursion relation for $R_{n}(\lambda)$ :

$$
\begin{aligned}
& \left(c_{1}^{(1)}-\lambda c_{1}^{(2)}\right) R_{1}+\left(b_{0}^{(1)}-\lambda b_{0}^{(2)}\right) R_{0}=0, \\
& \left(c_{n+1}^{(1)}-\lambda c_{n+1}^{(2)}\right) R_{n+1}+\left(b_{n}^{(1)}-\lambda b_{n}^{(2)}\right) R_{n}+\left(a_{n}^{(1)}-\lambda a_{n}^{(2)}\right) R_{n-1}=0, \quad n=1,2(2.4)
\end{aligned}
$$

In what follows we will assume that

$$
c_{n}^{(1)} c_{n}^{(2)} \neq 0, a_{n}^{(1)} a_{n}^{(2)} \neq 0 n=1,2, \ldots, N-1 .
$$

Then it is seen from (2.4) that

$$
R_{n}(z)=R_{0}(z) \frac{P_{n}(z)}{\left(z-\alpha_{1}\right)\left(z-\alpha_{2}\right) \ldots\left(z-\alpha_{n}\right)},
$$

where $P_{n}(z)$ is a polynomial of degree $\leq n, R_{0}(z)$ is an arbitrary function and

$$
\alpha_{n}=\frac{c_{n}^{(1)}}{c_{n}^{(2)}} \text {. }
$$

It is natural to put $R_{0}(z)=1$ (initial condition). Then from $(2.6) R_{n}(z)$ is a rational function in $z$ with the prescribed poles $\alpha_{k}, k=1,2, \ldots, n$. 
Consider conjugated GEVP (1.2), where in our case $A^{*}, B^{*}$ are transposed matrices, i.e.

$$
A^{*} e_{n}=c_{n+1}^{(1)} e_{n+1}+b_{n}^{(1)} e_{n}+a_{n}^{(1)} e_{n-1}, \quad B^{*} e_{n}=c_{n+1}^{(2)} e_{n+1}+b_{n}^{(2)} e_{n}+a_{n}^{(2)} e_{n-1}
$$

We can write the corresponding generalized eigenvector $\psi(\lambda)$ as

$$
\psi^{*}(\lambda)=\sum_{k=0}^{N-1} R_{k}^{*}(\lambda) e_{k} .
$$

Taking again the initial condition $R_{0}^{*}=1$ we arrive at the expression

$$
R_{n}^{*}(z)=\kappa_{n} \frac{P_{n}(z)}{\left(z-\beta_{1}\right)\left(z-\beta_{2}\right) \ldots\left(z-\beta_{n}\right)},
$$

where $P_{n}(z)$ is the same polynomial as in (2.6) and

$$
\beta_{n}=\frac{a_{n}^{(1)}}{a_{n}^{(2)}}, \quad \kappa_{n}=\prod_{k=1}^{n} \frac{c_{k}^{(2)}}{a_{k}^{(2)}} .
$$

Note that due to condition (2.5) we have $\kappa_{n} \neq 0, \infty$. We thus see that $R_{n}^{*}(z)$ is also a rational function with the same zeroes as $R_{n}(z)$ and with prescribed poles $\beta_{k}, k=$ $1,2, \ldots, n$.

The polynomials $P_{n}(z)$ play an important role in the theory of biorthogonal rational functions. They satisfy the three-term recurrence relation

$$
c_{n+1}^{(2)} P_{n+1}(z)+\left(z b_{n}^{(2)}-b_{n}^{(1)}\right) P_{n}(z)+a_{n}^{(2)}\left(z-\alpha_{n}\right)\left(z-\beta_{n}\right) P_{n-1}(z)=0
$$

which belongs to a class of so-called $R_{I I}$ recurrence relations considered in [10]. There is a relation of the polynomial $P_{n}(z)$ with the characteristic determinant $\Delta_{n}(z)$ of truncated matrices [20]:

$$
P_{n}(z)=\frac{\Delta_{n}(z)}{c_{1}^{(2)} c_{2}^{(2)} \ldots c_{n}^{(2)}}
$$

where

$$
\Delta_{n}(z)=\operatorname{det}\left(A_{n}-z B_{n}\right)
$$

and matrices $A_{n}, B_{n}$ are $n \times n$ matrices consisting of the first $n$ rows and columns of matrices $A, B$. In the finite-dimensional case we obtain the generalized eigenvalues $\lambda$ as roots of the characteristic polynomial

$$
P_{N}(z)=0 .
$$

In what follows we will assume that in the finite-dimensional case all $N$ roots of the polynomial $P_{N}(z)$ are simple. 
Now, using (1.5) we can construct the biorthogonal partner $T_{n}(z)$ for the function $R_{n}(z)$. In our case the biorthogonal vector $\psi(\lambda)$ is constructed as $\psi(\lambda)=B^{*} \psi^{*}(\lambda)$. Expanding the vector $\phi(z)$ as

$$
\phi(z)=\sum_{n=0}^{N-1} T_{n}(z) e_{n}
$$

we have from (1.5)

$$
\sum_{n=0}^{N-1} T_{n}(z) e_{n}=\sum_{n=0}^{N-1} R_{n}^{*}(z) B^{*} e_{n}
$$

and hence

$$
T_{n}(z)=a_{n+1}^{(2)} R_{n+1}^{*}(z)+b_{n}^{(2)} R_{n}^{*}(z)+c_{n}^{(2)} R_{n-1}^{*}(z) .
$$

Using the recurrence relation for the rational functions $R_{n}^{*}(z)$ we can rewrite the expression (2.16) in the equivalent forms:

$$
T_{n}(z)=\left(a_{n+1}^{(1)} R_{n+1}^{*}(z)+b_{n}^{(1)} R_{n}^{*}(z)+c_{n}^{(1)} R_{n-1}^{*}(z)\right) z^{-1}
$$

or

$$
T_{n}(z)=\frac{\left(a_{n+1}^{(1)} c_{n}^{(2)}-a_{n+1}^{(2)} c_{n}^{(1)}\right) R_{n+1}^{*}(z)+\left(b_{n}^{(1)} c_{n}^{(2)}-b_{n}^{(1)} c_{n}^{(2)}\right) R_{n}^{*}(z)}{z c_{n}^{(2)}-c_{n}^{(1)}}
$$

or

$$
T_{n}(z)=\frac{\left(a_{n+1}^{(2)} b_{n}^{(1)}-a_{n+1}^{(1)} b_{n}^{(2)}\right) R_{n}^{*}(z)+\left(a_{n+1}^{(2)} c_{n}^{(1)}-a_{n+1}^{(1)} c_{n}^{(2)}\right) R_{n-1}^{*}(z)}{z a_{n+1}^{(2)}-a_{n+1}^{(1)}}
$$

From (2.19) it is clear that $T_{n}(z)$ is a rational function of the form

$$
T_{n}(z)=\frac{Q_{n}(z)}{\left(z-\beta_{1}\right)\left(z-\beta_{2}\right) \ldots\left(z-\beta_{n+1}\right)},
$$

where $Q_{n}(z)$ is a polynomial with degree $\leq n$. We thus see that, in contrast to $R_{n}^{*}(z)$, the function $T_{n}(z)$ consists of $n+1$ poles, i.e it contains additional pole at $z=\beta_{n+1}$.

As was shown in [20] for rational functions $R_{n}(z)$ and $T_{n}(z)$ there exists a linear functional $\sigma$ (defined on the space of rational functions with prescribed poles $\alpha_{i}$ and $\beta_{i}$ ) such that

$$
\left.\sigma\left\{R_{n}(z) T_{m}(z)\right\}\right)=h_{n} \delta_{n m}
$$

with some nonzero normalization constants $h_{n}$. Property (2.21) means that rational functions $R_{n}(z), T_{m}(z)$ form two biorthogonal families. For the most general explicit biorthogonal rational functions expressible in terms of elliptic functions see [16], [17]. 


\section{Self-similar closure of the Darboux transformation and admissible poles}

In this section we consider simplest self-similar closures of the Darboux transformations for GEVP.

Assume that the transformed operators $\tilde{A}, \tilde{B}$ are expressed as linear combinations of the initial ones:

$$
\tilde{A}=\xi_{1} A+\xi_{2} B, \quad \tilde{B}=\xi_{3} A+\xi_{4} B
$$

where $\xi_{i}$ are some constants such that $\xi_{1} \xi_{4}-\xi_{2} \xi_{3} \neq 0$.

If condition (3.1) holds then the transformed GEVP can be presented in the form:

$$
0=(\tilde{A}-\lambda \tilde{B}) \tilde{\psi}(\lambda)
$$

or, equivalently,

$$
A \tilde{\psi}(\lambda)=\tilde{\lambda} B \tilde{\psi}(\lambda)
$$

where

$$
\tilde{\lambda}=\frac{\lambda \xi_{4}-\xi_{2}}{\xi_{1}-\lambda \xi_{3}} .
$$

From (3.3) and (1.1) we conclude that the vector $\tilde{\psi}(\lambda)$ belongs to the kernel space of the operator $A-\tilde{\lambda} B$. On the other hand, we know that $\tilde{\psi}(\lambda)=B \psi(\lambda)$. From this we can easily conclude that the expansion coefficients $\tilde{\psi}_{n}(\lambda)$ with respect to the basis $e_{n}$ can be presented as

$$
\tilde{\psi}_{n}(\lambda)=G(\lambda) \psi_{n}(\tilde{\lambda}), n=0,1, \ldots
$$

with some function $G(\lambda)$. More detailed consideration similar to that in the previous section yields

$$
(B \psi(z))_{n} \equiv \tilde{\psi}_{n}(\lambda)=\frac{Y_{n}(z)}{\left(z-\alpha_{1}\right)\left(z-\alpha_{2}\right) \ldots\left(z-\alpha_{n+1}\right)}
$$

where $Y_{n}(z)$ is a polynomial in $z$ of degree $\leq n$.

On the other hand, by (3.4) we have

$$
\tilde{\psi}_{n}(z)=\frac{G(z) P_{n}(\tilde{z})}{\left(\tilde{z}-\alpha_{1}\right)\left(\tilde{z}-\alpha_{2}\right) \ldots\left(\tilde{z}-\alpha_{n}\right)}=\frac{G(z) \tilde{P}_{n}(z)}{\left(z-\tilde{\alpha}_{1}\right)\left(z-\tilde{\alpha}_{2}\right) \ldots\left(z-\tilde{\alpha}_{n}\right)}
$$

where

$$
\tilde{P}_{n}(z)=\kappa_{n}\left(\xi_{1}-z \xi_{3}\right)^{n} P_{n}\left(\frac{z \xi_{4}-\xi_{2}}{\xi_{1}-z \xi_{3}}\right), \quad \kappa_{n}=\prod_{i=1}^{n}\left(\xi_{4}+\alpha_{i} \xi_{2}\right)
$$

and new poles $\tilde{\alpha}_{n}$ are

$$
\tilde{\alpha}_{n}=\frac{\alpha_{n} \xi_{1}+\xi_{2}}{\alpha_{n} \xi_{3}+\xi_{4}}
$$


We have an identity

$$
\frac{Y_{n}(z)}{\left(z-\alpha_{1}\right)\left(z-\alpha_{2}\right) \ldots\left(z-\alpha_{n+1}\right)}=\frac{G(z) \tilde{P}_{n}(z)}{\left(z-\tilde{\alpha}_{1}\right)\left(z-\tilde{\alpha}_{2}\right) \ldots\left(z-\tilde{\alpha}_{n}\right)}
$$

This identity should be valid for all $n=0,1, \ldots$ For $n=0$ we get (as $P_{0}=1$ and $Y_{0}(z)=Y_{0}=$ const $)$

$$
G(z)=\frac{Y_{0}}{z-\alpha_{1}}
$$

For all other $n$ we obtain the restriction upon poles $\alpha_{n}$ :

$$
\frac{\alpha_{n} \xi_{1}+\xi_{2}}{\alpha_{n} \xi_{3}+\xi_{4}}=\alpha_{n+1}
$$

This restriction can be considered as a discrete Riccati equation with constant coefficients. Its general solution can be found in an elementary way:

$$
\alpha_{n}=\frac{\mu_{1} q^{n}+\mu_{2}}{\mu_{3} q^{n}+\mu_{4}}
$$

with some constants $q, \mu_{i}$ or

$$
\alpha_{n}=\frac{\mu_{1} n+\mu_{2}}{\mu_{3} n+\mu_{4}}
$$

The solution (3.12) corresponds to the case when the matrix

$$
\Xi=\left(\begin{array}{ll}
\xi_{1} & \xi_{2} \\
\xi_{3} & \xi_{4}
\end{array}\right)
$$

is diagonalizable with distinct eigenvalues $\lambda_{1}, \lambda_{2}$, in this case $q=\lambda_{1} / \lambda_{2}$. Solution (3.13) corresponds to the case when the matrix $\Xi$ is non-diagonalizable.

Quite analogously we obtain similar expressions for poles $\beta_{n}$ :

$$
\beta_{n}=\frac{\nu_{1} q^{n}+\nu_{2}}{\nu_{3} q^{n}+\nu_{4}}
$$

with some constants $\nu_{i}$ or

$$
\beta_{n}=\frac{\nu_{1} n+\nu_{2}}{\nu_{3} n+\nu_{4}}
$$

We thus see that the rational functions $\psi_{n}(z)$ corresponding to GEVP with the closure condition (3.1) have the self-similarity property (3.4) with respect to action of the operator $B$, i.e. the corresponding Darboux transformation leads only to a rational transformation of the argument. Moreover, the poles of the rational functions have an explicit dependence on $n$ given by formulas (3.12), (3.14), or (3.13), (3.15). 


\section{Canonical forms of the closure condition and regular al- gebras of dimension 2}

Substituting the closure condition (3.1) into the Darboux relation (1.16) we obtain that operators $A, B$ satisfy an algebraic relation

$$
\left(\xi_{1} A+\xi_{2} B\right) B=\left(\xi_{3} A+\xi_{4} B\right) A
$$

This is a quadratic relation involving all second-degree combinations $A^{2}, B^{2}, A B, B A$.

We already know that the initial GEVP (1.1) preserves its form under arbitrary linear transformation (1.6) (with projective transformed eigenvalue $\lambda$ ). We can use this property to simplify our problem as much as possible.

Assume first that the matrix $\Xi$ is diagonalizable with distinct nonzero eigenvalues $q_{1}, q_{2}$, i.e. $\operatorname{tr}_{\Xi}^{2}-4 \Delta_{\Xi}=\left(\xi_{1}+\xi_{4}\right)^{2}-4\left(\xi_{1} \xi_{4}-\xi_{2} \xi_{3}\right) \neq 0$ (as usual, $\operatorname{tr}_{\Xi}, \Delta_{\Xi}$ are the trace and determinant of the matrix $\Xi$ ). Then it is possible to choose the linear transformation (1.6) such that

$$
\Xi=\left(\begin{array}{cc}
q_{1} & 0 \\
0 & q_{2}
\end{array}\right)
$$

In this case as easily seen we obtain instead of (4.1) the simple Weyl-type operator relation

$$
A B=q B A
$$

where $q=q_{2} / q_{1}$ (note that zero values of $q_{1}, q_{2}$ are forbidden by the restriction $\Delta_{\Xi} \neq 0$ ). If the matrix $\Xi$ is non-diagonalizable then it is possible to reduce it to (say) the form

$$
\Xi=\left(\begin{array}{cc}
1 & -1 \\
0 & 1
\end{array}\right)
$$

In this case algebraic relation (4.1) becomes

$$
A B-B A=B^{2}
$$

It is interesting to note that relations (4.2) and (4.3) describe two possible types of socalled regular algebras of dimension two [1], [8]. We thus see that these rather abstract objects as regular algebras appear naturally in our approach.

In what follows we will assume that $q$ is a real parameter, $q \neq 0, \pm 1$. In this case we can obtain only infinite-dimensional representations of the Weyl relation (4.2). Indeed, if $A, B$ are nondegenerated matrices of dimension $N$ (i.e. $\operatorname{det}(A) \operatorname{det}(B) \neq 0)$ then it follows from (4.2) that $q^{N}=1$. Thus nondegenerate finite-dimensional representations of the Weyl algebras are possible only for $q$ a root of unity.

Note that the Weyl relation (4.2) is well known in quantum mechanics; it lies also in the base of the theory of "quantum planes" and related quantum groups and algebras (see, e.g. [13]). Relation (4.3) is closely connected with so-called raising-lowering operators. Indeed, assume that two operators $L, M$ satisfy the simplest linear commutation relation $[L, M]=M$. The operator $M$ is called raising operator with respect to the operator $L$, because if $\psi(\lambda)$ is any eigenvector from eigenspace of the operator $L$ with eigenvalue $\lambda$, then $M \psi(\lambda)$ is again eigenvector of the operator $L$ but with the shifted eigenvalue $\lambda+1$. Now choosing $A=L M, B=M$ we see that relation (4.3) is fulfilled. 


\section{Realizations of the regular algebra. Type I}

Consider the commutation relation

$$
A B-B A=B^{2}
$$

We will assume that off-diagonal entries of the operators $A, B$ are non-zero (non-degeneracy condition). Note that any non-degenerate diagonal operator $S$ (i.e. $S e_{n}=s_{n} e_{n}$ with some nonzero $s_{n}$ ) can be used for the similarity transformation of the operators $A, B$ : $A \rightarrow S A S^{-1}, B \rightarrow S B S^{-1}$. Clearly, for such transformed operators relation (5.1) will still hold. We thus can choose the diagonal operator $S$ to simplify the coefficients of the operators $A, B$. In particular, it is possible to choose $S$ such that the operator $B$ will be symmetric in the basis $e_{n}$ :

$$
B e_{n}=a_{n+1} e_{n+1}+b_{n} e_{n}+a_{n} e_{n-1}
$$

with some coefficients $a_{n}, b_{n}$. By nondegeneracy condition we have $a_{n} \neq 0, n=1,2, \ldots$.

For the operator $A$ we take the general 3-diagonal form

$$
A e_{n}=d_{n+1} e_{n+1}+g_{n} e_{n}+r_{n} e_{n-1}
$$

with some coefficients $d_{n}, r_{n}, g_{n}$. We assume that $d_{n} r_{n} \neq 0, n=1,2, \ldots$ We thus have 5 unknown coefficients $a_{n}, b_{n}, d_{n}, e_{n}, r_{n}$. Substituting (5.3) and (5.2) into commutation relation (5.1) we obtain a system of equations for these coefficients. Equating terms in front of vectors $e_{n \pm 2}$ we get

$$
d_{n}=(n-n) a_{n}, \quad r_{n}=-\left(n-n_{1}\right) a_{n},
$$

where $n_{0}, n_{1}$ are arbitrary parameters. Equating terms in front of vectors $e_{n \pm 1}$ we get two equations

$$
g_{n}-g_{n-1}+\left(n-n_{0}\right)\left(b_{n-1}-b_{n}\right)=b_{n}+b_{n-1},
$$

and

$$
g_{n-1}-g_{n}-\left(n-n_{1}\right)\left(b_{n}-b_{n-1}\right)=b_{n}+b_{n-1},
$$

Solving these equations we obtain

$$
b_{n}=\frac{\beta}{\left(2 n-n_{0}-n_{1}\right)\left(2 n+2-n_{0}-n_{1}\right)}
$$

with some parameter $\beta$ and

$$
g_{n}=b_{n}\left(n_{0}-n_{1}\right) / 2+\gamma
$$

with some parameter $\gamma$. Finally, equating terms in front of diagonal vectors $e_{n}$, we get equation

$$
\mu_{n-1 / 2} a_{n}^{2}-a_{n+1}^{2} \mu_{n+3 / 2}=b_{n}^{2}
$$

where $\mu_{n}=2 n-n_{0}-n_{1}$. It is easily verified that general solution of (5.7) is

$$
a_{n}^{2}=\frac{\beta^{2}+4 \varepsilon\left(2 n-n_{0}-n_{1}\right)^{2}}{4\left(2 n-n_{0}-n_{1}\right)^{2}\left(2 n+1-n_{0}-n_{1}\right)\left(2 n-1-n_{0}-n_{1}\right)}
$$


where $\varepsilon$ is a parameter.

In what follows we will demand $a_{0}=0$. This conditions merely means that the representation is bounded from below by the vector $e_{0}$. From this condition we find that

$$
\varepsilon=-\frac{\beta^{2}}{4\left(n_{0}+n_{1}\right)^{2}}
$$

The GEVP

$$
(A-z B) \psi=0
$$

can be written as

$$
\left(d_{n}-z a_{n}\right) \psi_{n-1}+\left(g_{n}-z b_{n}\right) \psi_{n}+\left(r_{n+1}-z a_{n+1}\right) \psi_{n+1}=0
$$

or

$$
a_{n}\left(n-n_{0}-z\right) \psi_{n-1}+\left(g_{n}-z b_{n}\right) \psi_{n}+a_{n+1}\left(n_{1}-n-z\right) \psi_{n+1}=0
$$

This recurrence relation defines some rational function $\psi_{n}(z)$ up to an arbitrary factor $\psi_{0}(z)$. In order to find $\psi_{n}(z)$ we first renormalize functions $\psi_{n}(z)=\xi_{n}(z) P_{n}(z)$ where $\xi_{n}(z)$ is the denominator of $\psi_{n}(z)$. We then obtain:

$$
P_{n+1}(z)+B_{n}(z) P_{n}(z)+U_{n}(z) P_{n-1}(z)=0,
$$

where

$$
B_{n}(z)=\gamma+\frac{\beta\left(n_{0}-n_{1}+2 z\right)}{2\left(2 n-n_{0}-n_{1}\right)\left(2 n+2-n_{0}-n_{1}\right)}
$$

and

$$
U_{n}(z)=\frac{\beta^{2}}{\left(n_{0}+n_{1}\right)^{2}} \frac{n\left(n-n_{0}-n_{1}\right)\left(n-n_{0}-z\right)\left(n-n_{1}+z\right)}{\left(2 n-n_{0}-n_{1}\right)^{2}\left(2 n-1-n_{0}-n_{1}\right)\left(2 n+1-n_{0}-n_{1}\right)}
$$

The solution of this recurrence relation can be found if one notices that relation (5.13) formally coincides with the 3 -term recurrence relation for the Jacobi polynomials [11] (after appropriate redefinition of the argument and parameters of polynomials). Omitting technical details we present the result:

$$
P_{n}(z)=\left(\frac{\beta}{n_{0}+n_{1}}\right)^{n} \frac{\left(1-n_{1}+z\right)_{n}}{\left(1+n-n_{0}-n_{1}\right)_{n}}{ }_{2} F_{1}\left(\begin{array}{c}
-n, 1+n-n_{0}-n_{1} \\
1+z-n_{1}
\end{array} \mid 1 / 2+\gamma\left(n_{0}+n_{1}\right) / \beta\right)
$$

The corresponding rational functions $\psi_{n}(z)$ with initial conditions $\psi_{-1}=0, \psi_{0}=1$ are

$$
\psi_{n}(z)=\frac{\beta^{n}}{\left(n_{0}+n_{1}\right)^{n}\left(1+n-n_{0}-n_{1}\right)_{n}}{ }_{2} F_{1}\left(\begin{array}{c}
-n, 1+n-n_{0}-n_{1} \\
1+z-n_{1}
\end{array} \mid 1 / 2+\gamma\left(n_{0}+n_{1}\right) / \beta\right)
$$


Consider the adjacent problem. New vectors $\tilde{\psi}=B \psi$. We have

$$
\tilde{A} \tilde{\psi}=\lambda \tilde{B} \tilde{\psi}
$$

where $\tilde{A}=A-B, \tilde{B}=B$. Equivalently,

$$
A \tilde{\psi}=(\lambda+1) B \tilde{\psi}
$$

Thus the new function $\tilde{\psi}(z)$ differs from $\psi(z)$ by a shift of the argument:

$$
\tilde{\psi}_{n}(z)=\text { const } \psi_{n}(z+1)
$$

A direct check shows that indeed

$$
\tilde{\psi}(z) \equiv(B \psi)_{n}=\frac{\beta+2 \gamma\left(n_{0}+n_{1}\right)}{2\left(n_{0}+n_{1}\right)\left(z+1-n_{1}\right)} \psi_{n}(z+1)
$$

\section{Moments and orthogonality}

In the previous section we constructed rational functions $\psi_{n}(z)$ as eigensolutions of GEVP. Omitting unnecessary factors we can put

$$
U_{n}(z)={ }_{2} F_{1}\left(\begin{array}{c}
-n, 1+n-n_{0}-n_{1} \mid \mu \\
1+z-n_{1}
\end{array}\right),
$$

where $\mu=1 / 2+\gamma\left(n_{0}+n_{1}\right) / \beta$. Elementary calculations from the general scheme (1.5) show that their biorthogonal partners $V_{n}(z)$ can be chosen as

$$
V_{n}(z)={ }_{2} F_{1}\left(\begin{array}{c}
-n, 1+n-n_{0}-n_{1} \\
2-n_{0}-z
\end{array} \mid 1-\mu\right)
$$

In this section we derive directly biorthogonality property of the rational functions $U_{n}(z), V_{n}(z)$.

The rational functions $U_{n}(z), V_{n}(z)$ can be presented in terms of partial fractions:

$$
U_{n}(z)=\xi_{n 0}+\sum_{k=1}^{n} \frac{\xi_{n k}}{z-a_{k}}, \quad V_{n}(z)=\eta_{n 0}+\sum_{k=1}^{n} \frac{\eta_{n k}}{z-b_{k}}
$$

where $a_{k}=n_{1}-k, b_{k}=k+1-n_{0}$ are the locations of poles. However, for our purposes it is more convenient to present functions $U_{n}(z), V_{n}(z)$ as the following decomposition

$$
U_{n}(z)=\sum_{k=0}^{n} \frac{\xi_{n k}}{\left(z-n_{1}+1\right)_{k}}, \quad V_{n}(z)=\sum_{k=0}^{n} \frac{\eta_{n k}}{\left(z-k-1+n_{0}\right)_{k}}
$$

Introduce a functional $\sigma$ defined on rational functions with poles $a_{k}, b_{k}$ by the formula

$$
\sigma_{n m} \equiv \sigma\left\{\frac{1}{\left(z-n_{1}+1\right)_{n}\left(z-m-1+n_{0}\right)_{m}}\right\}=(-1)^{m} \frac{\mu^{-n}(1-\mu)^{-m}}{\left(2-n_{0}-n_{1}\right)_{n+m}}
$$

This functional satisfies the restriction

$$
\sigma_{n-1, m}-\sigma_{n, m-1}=\left(n+m-n_{0}-n_{1}+1\right) \sigma_{n, m}
$$

It is easily verified that this restriction is equivalent to compatibility condition for a matrix $\sigma_{n, m}$ to be a moment matrix on the space of rational functions with prescribed poles $a_{k}, b_{k}$. 
Theorem 1. The rational functions $U_{n}(z), V_{n}(z)$ are biorthogonal with respect to the functional $\sigma$ :

$$
\sigma\left\{U_{n}(z) V_{m}(z)\right\}=h_{n} \delta_{n m}
$$

where

$$
h_{n}=\frac{(-1)^{n} n !}{\left(1+2 n-n_{0}-n_{1}\right)\left(2-n_{0}-n_{1}\right)_{n-1}}
$$

Proof. It is sufficient to present both $U_{n}(z), V_{m}(z)$ as hypergeometric series and then apply the functional defined by (6.5):

$$
\sigma\left\{U_{n}(z) V_{m}(z)\right\}=\sum_{k, s=0}^{N} \frac{(-n)_{k}(-m)_{s}\left(1+n-n_{0}-n_{1}\right)_{k}\left(1+m-n_{0}-n_{1}\right)_{s}}{k ! s !\left(2-n_{0}-n_{1}\right)_{k}\left(2-n_{0}-n_{1}+k\right)_{s}}
$$

where $N$ is any positive integer such that $N>\min (n, m)$. Then we can calculate the sum with respect to $s$ reducing it to the hypergeometric function ${ }_{2} F_{1}$ from unit argument which can be further simplified to get

$$
\sigma\left\{U_{n}(z) V_{m}(z)\right\}=\sum_{k=m}^{n} \frac{(-n)_{k}\left(1+n-n_{0}-n_{1}\right)_{k}(1+k-m)_{m}}{k !\left(2-n_{0}-n_{1}\right)_{k}\left(2-n_{0}-n_{1}+k\right)_{k}}
$$

This expression is 0 if $m>n$. But due to obvious symmetry between $n, m$ in (6.8) we can conclude that this expression is 0 also for $n>m$. Thus we need only to check the case $n=m$. But in this case only one term in (6.9) survives, and we get (6.7).

\section{Padé interpolation of the exponential function}

The biorthogonal functions, obtained in the previous section, can be presented as

$$
U_{n}(z)=\frac{(-1)^{n}\left(1+n-n_{0}-n_{1}\right)_{n}}{\left(1+z-n_{1}\right)_{n}} P_{n}(z), \quad V_{n}(z)=\frac{(\mu-1)^{n}\left(1+n-n_{0}-n_{1}\right)_{n}}{\left(2-z-n_{0}\right)_{n}} P_{n}^{*}(z)
$$

where

$$
P_{n}(z)={ }_{2} F_{1}\left(\begin{array}{c}
-n,-n+n_{1}-z \\
-2 n+n_{0}+n_{1}
\end{array} \mid 1 / \mu\right)
$$

and

$$
P_{n}^{*}(z)={ }_{2} F_{1}\left(\begin{array}{c}
-n,-1-n+n_{0}+z \\
-2 n+n_{0}+n_{1}
\end{array} \mid 1 /(1-\mu)\right)
$$

Assume that the parameters $n_{0}, n_{1}$ satisfy the condition

$$
n_{0}+n_{1}=-j
$$


where $j$ is a positive integer. Introduce polynomials

$$
Q_{n+j}(z)={ }_{2} F_{1}\left(\begin{array}{c}
-n-j,-n+n_{1}-z \\
-2 n-j
\end{array} \mid 1 /(1-\mu)\right)
$$

of degree $n+j$. Then from the Pfaff-Euler transformation formula for the Gauss hypergeometric function [5] we get

$$
\frac{Q_{n+j}\left(z_{s}\right)}{P_{n}\left(z_{s}\right)}=(\mu /(\mu-1))^{s}, \quad s=0,1, \ldots, 2 n+j
$$

where the grid is

$$
z_{s}=s-n+n_{1}
$$

Analogously

$$
\frac{Q_{n+j}^{*}\left(y_{s}\right)}{P_{n}^{*}\left(y_{s}\right)}=(1-1 / \mu)^{s}, \quad s=0,1, \ldots, 2 n+j
$$

where

$$
y_{s}=1+n-n_{0}-s
$$

We see that the obtained polynomials solve the Padé interpolation problem (for arbitrary positive integer $j$ ) for the exponential function $f(z)=\exp (\omega z)$ on linear grids.

The Padé interpolation table for the exponential function on the uniform was first explicitly constructed by Iserles [9]. See also [22] for further analysis and relations with theory of orthogonal polynomials and biorthogonal rational functions.

\section{The Weyl algebra representations}

Consider the Weyl commutation relation

$$
A B=q B A
$$

We are seeking a realization of this relation by three-term operators. In this case it is convenient to choose the similarity transformation $S$ such that the operator $A$ has unit coefficient in front of $e_{n-1}$, whereas $B$ is arbitrary 3-diagonal:

$$
A e_{n}=e_{n-1}+b_{n} e_{n}+u_{n+1} e_{n+1}, \quad B e_{n}=\rho_{n} e_{n-1}+g_{n} e_{n}+d_{n+1} e_{n+1}
$$

Note that this problem was already solved in our paper [19], where, however, we did not consider relation with GEVP.

From the commutation relation (8.1) we easily find

$$
d_{n}=c_{1} q^{-n} u_{n}, \quad \rho_{n}=c_{2} q^{n}
$$

where $c_{1}, c_{2}$ - arbitrary constants. Moreover

$$
b_{n}=-\frac{c_{3}\left(c_{1} q^{1-n}+c_{2} q^{n+2}+c_{4}\right)}{w_{n} w_{n-1}}
$$


where

$$
w_{n}=c_{1} q^{-n}-c_{2} q^{n+2}
$$

and $c_{4}$ is an arbitrary.

$$
g_{n}=\frac{b_{n}\left(c_{1} q^{-n}+c_{2} q^{n+1}\right)+c_{3}}{q+1}
$$

Finally, for $u_{n}$ we have the expression

$$
u_{n}=\frac{w_{n} w_{n-2} b_{n} b_{n-1}+c_{5}}{(q+1)^{2} v_{n} v_{n-1}}
$$

where

$$
v_{n}=c_{1} q^{-n}-c_{2} q^{n+1}
$$

From the condition $u_{0}=0$ we get the restriction for the constants:

$$
c_{5}=-\frac{c_{3}^{2}\left(q s_{1}+s_{2}\right)\left(q s_{2}+s_{1}\right)}{\left(q(q+1)\left(c_{1}-c_{2}\right)\right)^{2}}
$$

where

$$
s_{1}=c_{4}+q(q+1) c_{1}, \quad s_{2}=c_{4}+q(q+1) c_{2}
$$

In this case it is possible to present $u_{n}$ in the form

$$
u_{n}=C q^{2 n-3} \frac{\left(1-q^{n}\right)\left(1-c_{2} q^{n} / c_{1}\right)\left(1+s_{2} q^{n} / s_{1}\right)\left(1+s_{1} c_{2} q^{n} /\left(s_{2} c_{1}\right)\right)}{\left(1-c_{2} q^{2 n} / c_{1}\right)^{2}\left(1-c_{2} q^{2 n+1} / c_{1}\right)\left(1-c_{2} q^{2 n-1} / c_{1}\right)},
$$

where $C=-\frac{c_{3}^{2} s_{1} s_{2}}{\left((q+1)\left(c_{1}-c_{2}\right) c_{1}\right)^{2}}$. (It is assumed that $\left.c_{1} \neq c_{2}\right)$.

Now the $\operatorname{GEVP}(A-z B) \psi=0$ for the vector $\psi=\sum_{n=0}^{\infty} \psi_{n}(z) e_{n}$ is reduced to solution of the three-term recurrence relation

$$
\left(1-z \rho_{n+1}\right) \psi_{n+1}(z)+\left(b_{n}-z g_{n}\right) \psi_{n}(z)+u_{n}\left(1-z d_{n}\right) \psi_{n-1}(z)=0
$$

with the initial conditions

$$
\psi_{-1}=0, \psi_{0}=1
$$

Renormalizing $\psi_{n}(z)=P_{n}(z) / D_{n}(z)$ where $D_{n}(z)$ is denominator of $\psi_{n}(z)$ and then identifying the recurrence relation $P_{n}(z)$ with that for big q-Jacobi polynomials [11] we get solution

$$
\psi_{n}(z)=\frac{\left(-q \frac{s_{1} c_{2}}{s_{2} c_{1}} ; q\right)_{n}}{\left(\frac{c_{2} q^{n+1}}{c_{1}} ; q\right)_{n}}{ }_{3} \Phi_{2}\left(\begin{array}{c}
q^{-n}, c_{2} q^{n+1} / c_{1}, 0 \\
-c_{2} s_{1} q /\left(c_{1} s_{2}\right), z c_{2}
\end{array} ; q ; q\right)
$$

Note that $\psi_{n}(z)$ is a rational function of order $[n / n]$ having poles $z=c_{2}^{-1} q^{-k}, k=$ $0,1, \ldots, n-1$. 
Consider the adjacent problem for the function $\tilde{\psi}=B \psi$. From the Weyl commutation relation it follows

$$
A \tilde{\psi}=\lambda q B \tilde{\psi}
$$

Hence

$$
\tilde{\psi}_{n}(z)=\text { const } \psi_{n}(q z)
$$

Consider the conjugated problem

$$
A^{*} \psi^{*}=z B^{*} \psi^{*}
$$

where

$$
A^{*} e_{n}=e_{n+1}+b_{n} e_{n}+u_{n} e_{n-1}, \quad B^{*} e_{n}=\rho_{n+1} e_{n+1}+g_{n} e_{n}+d_{n} e_{n-1}
$$

We have

$$
\psi_{n}^{*}(z)=\kappa_{n 3} \Phi_{2}\left(\begin{array}{c}
q^{-n}, c_{2} q^{n+1} / c_{1}, 0 \\
-s_{2} q / s_{1}, q^{2} /\left(z c_{1}\right)
\end{array} ; q ; q\right)
$$

where

$$
\kappa_{n}=q^{-n(n-1) / 2}\left(\left(c_{2}-c_{1}\right) c_{1} c_{3}(q+1) / s_{2}\right)^{n} \frac{\left(c_{2} q^{2} / c_{1} ; q\right)_{2 n}}{(q ; q)_{n}\left(-q c_{2} s_{1} /\left(c_{1} s_{2}\right) ; q\right)_{n}}
$$

It is sufficient to note that the biorthogonal partners

$$
\phi_{n}^{*} \equiv B^{*} \psi_{n}^{*}
$$

correspond the same GEVP

$$
A^{*} \phi^{*}=\lambda q^{-1} B^{*} \phi^{*}
$$

with rescaled eigenvalue parameter $\lambda \rightarrow \lambda / q$. Thus we have explicitly

$$
\phi_{n}^{*}(z)=\psi_{n}^{*}(z / q)=\kappa_{n 3} \Phi_{2}\left(\begin{array}{c}
q^{-n}, c_{2} q^{n+1} / c_{1}, 0 \\
-s_{2} q / s_{1}, q^{3} /\left(z c_{1}\right)
\end{array} ; q ; q\right)
$$

The rational function $\phi_{n}^{*}(z)$ has poles at points $z=c_{1}^{-1} q^{k+2}$. Consider the elementary rational functions

$$
\chi_{n}^{(1)}(z)=1 /\left(z c_{2} ; q\right)_{n}, \quad \chi_{n}^{(2)}(z)=1 /\left(q^{3} /\left(z c_{1}\right) ; q\right)_{n}
$$

Clearly, $\chi_{n}^{(1)}(z)$ has poles at $z=c_{2}^{-1}, \ldots, c_{2}^{-1} q^{1-n}$ and $\chi_{n}^{(2)}(z)$ has poles at $z=c_{1}^{-1} q^{3}, \ldots, c_{1}^{-1} q^{n+2}$, thus coincide with poles of $\psi_{n}(z), \phi_{n}^{*}(z)$

Define the matrix of "moments" for some linear functional $\sigma$ acting on the space of rational functions with these poles:

$$
\sigma_{n m}=\sigma\left\{\chi_{n}^{(1)}(z) \chi_{m}^{(2)}(z)\right\}=\frac{\left(-c_{2} s_{1} q /\left(c_{1} s_{2}\right) ; q\right)_{n}\left(-q s_{2} / s_{1} ; q\right)_{m}}{\left(q^{2} c_{2} / c_{1} ; q\right)_{n+m}}
$$

This functional satisfies the restriction

$$
\sigma_{n-1, m}+\sigma_{n, m-1}=\sigma_{n-1, m-1}+\left(1-q^{n+m+1}\right) \sigma_{n, m}
$$


which is equivalent to the compatibility condition for the matrix $\sigma_{n m}$ be a moment matrix for a linear functional defined on the space of rational functions with prescribed poles.

Instead of $\psi_{n}(z), \phi_{n}(z)$ we define now rational functions $U_{n}(z), V_{n}(z)$ which are essentially basic hypergeometric functions without unnecessary factors, i.e.

$$
U_{n}(z)={ }_{3} \Phi_{2}\left(\begin{array}{c}
q^{-n}, c_{2} q^{n+1} / c_{1}, 0 \\
-c_{2} s_{1} q /\left(c_{1} s_{2}\right), z c_{2}
\end{array} ; q ; q\right)
$$

and

$$
V_{n}(z)={ }_{3} \Phi_{2}\left(\begin{array}{c}
q^{-n}, c_{2} q^{n+1} / c_{1}, 0 \\
-s_{2} q / s_{1}, q^{3} /\left(z c_{1}\right)
\end{array} ; q ; q\right)
$$

Theorem 2. Functions $U_{n}(z), V_{m}(z)$ are biorthogonal with respect to functional $\sigma$ :

$$
\sigma\left\{U_{n}(z) V_{m}(z)\right\}=h_{n} \delta_{n m}
$$

where

$$
h_{n}=\frac{(-1)^{n}\left(c_{2} / c_{1}\right)^{n} q^{n(n+3) / 2}(q ; q)_{n}}{\left(c_{2} q^{2} / c_{1} ; q\right)_{n-1}\left(1-c_{2} q^{2 n+1} / c_{1}\right)}
$$

Proof. Expanding functions $U_{n}(z), V_{m}(z)$ in terms of $\chi_{i}^{(1)}(z), \chi_{k}^{(2)}$ and substituting to lhs of (8.23) we get the double sum

$$
\sigma\left\{U_{n}(z) V_{m}(z)\right\}=\sum_{i k=0}^{N} \frac{\left(q^{-n} ; q\right)_{i}\left(c_{2} q^{n+1} / c_{1} ; q\right)_{i}\left(q^{-m} ; q\right)_{k}\left(c_{2} q^{m+1} / c_{1} ; q\right)_{k}}{(q ; q)_{i}(q ; q)_{k}\left(c_{2} q^{2} / c_{1} ; q\right)_{i+k}} q^{i+k}
$$

where $N \geq \min (n, m)$.

Taking into account that

$$
\left(c_{2} q^{2} / c_{1} ; q\right)_{i+k}=\left(c_{2} q^{2} / c_{1} ; q\right)_{i}\left(c_{2} q^{2+i} / c_{1} ; q\right)_{k}
$$

we can separate summation in $k$ and $i$ :

$$
\begin{aligned}
& \sigma\left\{U_{n}(z) V_{m}(z)\right\}=\sum_{i=0}^{n} \frac{\left(q^{-n} ; q\right)_{i}\left(c_{2} q^{n+1} / c_{1} ; q\right)_{i}}{(q ; q)_{i}\left(c_{2} q^{2} / c_{1} ; q\right)_{i}} q^{i} \times \\
& \sum_{k=0}^{m} \frac{\left(q^{-m} ; q\right)_{k}\left(c_{2} q^{m+1} / c_{1} ; q\right)_{k}}{(q ; q)_{k}\left(c_{2} q^{2+i} / c_{1} ; q\right)_{k}} q^{k}
\end{aligned}
$$

The sum in $k$ can be calculated using the standard summation formula for basic hypergeometric function [7], [11]:

$$
\sum_{k=0}^{m} \frac{\left(q^{-m} ; q\right)_{k}\left(c_{2} q^{m+1} / c_{1} ; q\right)_{k}}{(q ; q)_{k}\left(c_{2} q^{2+i} / c_{1} ; q\right)_{k}} q^{k}=\frac{q^{m(m+1)}\left(c_{2} / c_{1}\right)^{m}\left(q^{1+i-m} ; q\right)_{m}}{\left(c_{2} q^{2+i} / c_{1} ; q\right)_{m}}
$$

The remaining expression contains term $\left(q^{1+i-m} ; q\right)_{m}$ which vanishes if $n<m$, hence $\sigma\left\{U_{n}(z) V_{m}(z)\right\}=0$ if $n<m$. But expression (8.25) is symmetric with respect to $n, m$, hence also $\sigma\left\{U_{n}(z) V_{m}(z)\right\}=0$ if $m<n$. We thus proved that functions $U_{n}(z)$ and $V_{m}(z)$ are biorthogonal for noncoinciding values of $n, m$. If $m=n$ then only one term (with $i=n$ ) survives in the sum (8.26) and we arrive at expression (8.24) for normalization coefficient $h_{n}$. 


\section{Interpolation of the power function}

Using transformation formulas for basic hypergeometric functions, we can present the rational functions $U_{n}(z)$ in the form

$$
\begin{aligned}
& U_{n}(z)=\left(-p s_{1} c_{2} z / s_{2}\right)^{-n} \frac{\left(c_{1} / c_{2} p^{n+1} ; p\right)_{n}}{\left(-c_{1} s_{2} p /\left(c_{2} s_{1}\right) ; p\right)_{n}\left(1 /\left(z c_{2}\right) ; p\right)_{n}} \times \\
& { }_{3} \Phi_{2}\left(\begin{array}{c}
\left.p^{-n},-c_{2} s_{1} p^{-n} /\left(c_{1} s_{2}\right), z c_{2} p^{1-n} ; p ; p\right) \\
0, c_{2} p^{-2 n} / c_{1}
\end{array}\right.
\end{aligned}
$$

where $p=1 / q$. Introduce the polynomials

$$
P_{n}(z)={ }_{3} \Phi_{2}\left(\begin{array}{c}
p^{-n},-c_{2} s_{1} p^{-n} /\left(c_{1} s_{2}\right), z c_{2} p^{1-n} ; p ; p \\
0, c_{2} p^{-2 n} / c_{1}
\end{array}\right)
$$

It is clear that (to within factor not depending on $z$ ) $P_{n}(z)$ are numerators of rational functions $U_{n}(z)$.

In what follows we assume that

$$
c_{2}=c_{1} q^{j}
$$

where $j$ is an arbitrary positive integer. Introduce polynomials

$$
Q_{n+j}(z)={ }_{3} \Phi_{2}\left(\begin{array}{c}
p^{-j-n},-s_{2} p^{-n} /\left(s_{1}\right), z c_{2} p^{1-n} \\
0, p^{-j-2 n}
\end{array} ; p ; p\right)
$$

having degree $n+j$ in $z$.

Using standard transformation formulas for the basic hypergeometric function [11] we easily find that

$$
\frac{P_{n}\left(z_{k}\right)}{Q_{n+j}\left(z_{k}\right)}=\left(-s_{2} / s_{1}\right)^{k}, \quad k=0,1, \ldots, 2 n+j
$$

where

$$
z_{k}=c_{2}^{-1} p^{-k+n-1}
$$

This is equivalent to Padé interpolation of the power function

$$
f(z)=z^{\alpha}
$$

where $\alpha$ is determined by

$$
p^{-\alpha}=-s_{2} / s_{1}
$$

Note that the Padé interpolation table for the exponential and power functions was constructed and analyzed in [22]. Our present interpolation problem differs from that of [22] by a shift of the interpolation grid, i.e. in [22] the initial point $z_{0}$ of interpolation grid was the same for all Padé entries $m, n$, whereas in the present case $z_{0}$ depends on $n, m$. 


\section{Realization of the moment functionals in terms of Fourier and Mellin integrals and the general interpolation prob- lem}

We defined the moment functionals $\sigma$ formally by the expressions (6.5) and (8.20). A natural question arises: is it possible to realize these functionals in terms of definite integrals with respect to some weight function? Such a realization is well known in the theory of orthogonal polynomials where the Favard theorem provides existence of such a representation (at least in terms of the Stieltjes integral, including purely discrete weights) [4].

For the generic situation we didn't find a corresponding representation. However such a representation does exist for the case of the Padé interpolation of the exponential and power functions, i.e. when we have restrictions (7.4) and (9.3).

Consider first the case of restriction (7.4). Note that shifting the parameter $n_{1}$ is equivalent to shifting the argument $z$. Hence we can put $n_{1}=0$ without loss of generality. Then $n_{0}=-j$ with some positive integer $j$.

In this case it is not difficult to show that the moments (6.5) can be presented as

$$
\sigma_{n m} \equiv \sigma\left\{\frac{1}{(z+1)_{n}(z-m-1-j)_{m}}\right\}=\frac{c_{j}}{2 \pi i} \int_{\Gamma} \frac{\exp (\omega \zeta) d \zeta}{W_{j}(\zeta)(\zeta+1)_{n}(\zeta-m-1-j)_{m}}
$$

where $e^{\omega}=\mu /(\mu-1), c_{j}=(j+1) !(\mu-1)^{j+1}$ and

$$
W_{j}(z)=z(z-1) \ldots(z-j-1)
$$

The contour $\Gamma$ is chosen such that all poles $z_{s}=s, s=-n,-n+1, \ldots, m+j+1$ coming from denominator in (10.1) lie inside the contour. Indeed, the integral in (10.1) is reduced to the sum of residues at $m+n+j+2$ poles $z_{s}=s, s=-n,-n+1, \ldots, m+j+1$. This sum, in turn, can be easily calculated using binomial theorem.

Now we can deform the contour $\Gamma$ transforming it to a vertical line. If $\omega<0$, we can choose this line passing to the left of the point $z=-n$, if $\omega>0$ we choose the line to the right of the point $z=m+j+1$. Indeed, by the Cauchy theorem, in these cases it is possible to close the contour adding a big semicircle with vanishing contribution to the integral.

We thus have

Theorem 3. . Assume that $n_{1}=0, n_{0}=-j$. Then the moment functional (6.5) can be presented in terms of the Fourier integral

$$
\sigma_{n m}=\frac{c_{j}}{2 \pi i} \int_{\alpha-i \infty}^{\alpha+i \infty} \frac{e^{\omega x} d x}{W_{j}(x)(x+1)_{n}(x-m-1-j)_{m}}
$$

where $\alpha<-n$ for $\omega<0$ and $\alpha>m+j+1$ for $\omega>0$.

Consider now the case of the moment functional (8.20) with an additional restriction

$$
c_{1}=q^{1-j}, c_{2}=q
$$


with some positive integer $j$. This restriction is equivalent to restriction (9.3) necessary for the Padé interpolation of the power function (the constant $c_{2}$ can be chosen arbitrarily without loss of generality because this leads merely to a shift of the interpolation grid $\left.z_{s}\right)$. In this case we have that the basic rational functions $\chi_{n}^{(1)}(z)=1 /(z q ; q)_{n}$ have the poles $z=q^{-1}, q^{-2}, \ldots, q^{-n}$ and the functions $\chi_{m}^{(2)}(z)=1 /\left(q^{j+2} / z ; q\right)_{m}$ have the poles $z=q^{j+2}, q^{j+3}, \ldots q^{j+m+1}$. Introduce the polynomial $W_{j}(z)=(z-1)(z-q) \ldots\left(z-q^{j+1}\right)$ of degree $j+2$. We then obtain that the moment functional (8.20) has the integral representation

$$
\sigma_{n m} \equiv \sigma\left\{\frac{1}{(z q ; q)_{n}\left(q^{j+2} / z ; q\right)_{m}}\right\}=\frac{\kappa_{j}}{2 \pi i} \int_{\Gamma} \frac{\zeta^{p} d \zeta}{W_{j}(\zeta)(\zeta q ; q)_{n}\left(q^{j+2} / \zeta ; q\right)_{m}}
$$

where

$$
q^{p}=-s_{2} / s_{1}, \kappa_{j}=\left(s_{1} / s_{2}\right)^{j+1} q^{j(j+1) / 2} \frac{(q ; q)_{j+1}}{\left(-s_{1} / s_{2} ; q\right)_{j+1}}
$$

and the contour $\Gamma$ is chosen to encircle all poles $z_{s}=q^{s}, s=-n, n+1, \ldots, m+j+1$. Verification of formula (10.4) is elementary using the Cauchy theorem and the q-binomial formula [11].

Assume now that additionally we have the restriction $-1<p<j+1$. Then the contour $\Gamma$ can be transformed into the contour consisting from two sides of negative real axis and two circles with the origin $z=0$ : one is very small and second is very large. The contribution from the circles tends to zero and we have

Theorem 4. Under the restrictions $c_{1}=q^{1-j}, c_{2}=q,-1<p<j+1$ we have representation of the moment functional (10.4) in terms of the Mellin integral

$$
\sigma_{n m}=\frac{\kappa_{j} \sin (\pi p)}{\pi} \int_{-\infty}^{0} \frac{|x|^{p} d x}{W_{j}(x)(x q ; q)_{n}\left(q^{j+2} / x ; q\right)_{m}}
$$

Now we can apply these results to biorthogonal rational functions corresponding to Padé interpolation of the exponential and power functions.

We first need a general result concerning biorthogonality of rational functions corresponding to general Padé interpolation scheme [23].

Let $F(z)$ be a function and $y_{s}, s=0, \pm 1, \pm 2, \ldots$ an infinite sequence of distinct points (the interpolation grid). We are seeking polynomials $Q_{n+M}(z ; M, k), P_{n}(z ; M, k)$ satisfying the interpolation property

$$
F\left(y_{s}\right)=\frac{Q_{n+M}\left(y_{s} ; M, k\right)}{P_{n}\left(y_{s} ; M, k\right)}, \quad s=-n+k,-n+k+1, \ldots, n+M+k
$$

We assume that the Padé scheme is nondegenerate, i.e. polynomials $Q_{n+M}(z ; M, k), P_{n}(z ; M, k)$ exist, have the degrees $n+M$ and $n$ and have no coinciding roots. The last condition is equivalent to the condition that roots of polynomials $Q_{n+M}(z ; M, k), P_{n}(z ; M, k)$ do not coincide with the interpolation grid $y_{s}[3]$.

It is convenient to normalize polynomials $P_{n}\left(y_{s} ; M, k\right)=z^{n}+O\left(z^{n-1}\right)$ to be monic (i.e. with highest-order term being unity). The polynomials $Q_{n+M}(z ; M, k)$ are not monic and have the expansion

$$
Q_{n+M}(z ; M, k)=\alpha_{n, n+M}(k) z^{n+M}+O\left(z^{n+M-1}\right)
$$


with some leading term $\alpha_{n, n+M}(k)$ depending on 3 integer parameters: $n, M, k$.

The additional integer parameter $k$ describes the shift of the interpolation grid. For $k=n$ we return to the standard interpolation scheme on the grid $y_{0}, y_{1}, \ldots, y_{2 n+M}$. In what follows we will consider the scheme with fixed value of the shift $k$. We will also fix the value of the parameter $M>0$ which means that we deal with a string from the Padé table parallel to the main diagonal $n=m$ (i.e. $M=0$ ).

Introduce polynomials

$$
W_{k M}(z)=\left(z-y_{k}\right)\left(z-y_{k+1}\right) \ldots\left(z-y_{M+k+1}\right)
$$

of degree $M+2$ and a pair of rational functions $U_{n}(z ; M, k)$ and $V_{n}(z ; n ; M, k)$ defined as

$$
\begin{aligned}
U_{n}(z ; M, k) & =\frac{P_{n}(z ; M, k)}{\left(z-y_{-n+k}\right)\left(z-y_{-n+k+1}\right) \ldots\left(z-y_{k-1}\right)} \\
V_{n}(z ; M, k) & =\frac{P_{n}(z ; M, k+1)}{\left(z-y_{M+2+k}\right)\left(z-y_{M+3+k}\right) \ldots\left(z-y_{n+M+k+1}\right)}
\end{aligned}
$$

Note that both rational functions $U_{n}(z ; M, k), V_{n}(z, M, k)$ have the type $[n / n]$ because the scheme is assumed to be nondegenerate and hence the roots of numerator polynomials in (10.8) cannot coincide with roots of denominators.

We then have

Theorem 5. The rational functions $U_{n}(z ; M, k), V_{n}(z, M, k)$ are biorthogonal with respect to the scalar product

$$
\frac{1}{2 \pi i} \int_{\Gamma} \frac{F(\zeta) U_{n}(\zeta ; M, k) V_{m}(\zeta ; M, k) d \zeta}{W_{k M}(\zeta)}=h_{n}(M, k) \delta_{n, m}
$$

or, equivalently,

$$
\left.\sum_{s=-n+k}^{m+M+k+1} F\left(y_{s}\right) \operatorname{Res}\left\{\frac{U_{n}(z ; M, k) V_{m}(z ; M, k)}{W_{k M}(z)}\right\}\right|_{z=y_{s}}=h_{n}(M, k) \delta_{n, m}
$$

where the normalization coefficients are

$$
h_{n}(M, k)=\frac{\alpha_{n, n+M+1}(k) \alpha_{n-1, n+M}(k)}{\alpha_{n-1, n+M}(k)-\alpha_{n, n+M+1}(k)}
$$

Remark. The contour $\Gamma$ in (10.9) is chosen to encircle all poles $z=y_{-n+k}, y_{-n+k+1}, \ldots y_{m+M+k+1}$ coming from all denominators and to avoid all singularity points of the function $F(z)$. In case if the function $F(z)$ is multi-valued, we should choose a regularity area in the complex plane with an appropriate system of cuts, all interpolation points $y_{s}$ and the contour $\Gamma$ should belong to the same area of regularity.

For the proof of this theorem see [23]. We mention only that this theorem is a simple consequence of the well known orthogonality property of the Padé interpolants with respect to a functional defined in terms of divided differences [12]. Nevertheless, as far as we know, explicit biorthogonality property (10.9) or (10.10) is a new result. (For the special case $k=n$ this theorem was proven in [17]). Note, that numerators $P_{n}(z ; M, k+1)$ of 
biorthogonal partners $V_{n}(z ; M, k)$ coincide with the numerator Padé interpolants for the same function $F(z)$ but on shifted interpolation grid (i.e. $k \rightarrow k+1$ ).

We now can apply this theorem to the Padé interpolation of the exponential and power functions.

Assume that we have exponential function $F(z)=e^{\omega z}$ with $\omega<0$. Assume also that all interpolation grids $y_{s}$ are real. Then from Theorem $\mathbf{5}$ we obtain that the contour $\Gamma$ can be transformed to a vertical line in the complex plane:

$$
\frac{1}{2 \pi i} \int_{\gamma-i \infty}^{\gamma+i \infty} \frac{\exp (\omega x) U_{n}(x ; M, k) V_{m}(x ; M, k) d \zeta}{W_{k M}(x)}=h_{n}(M, k), \delta_{n, m}
$$

where $\gamma$ is an arbitrary real parameter such that $\gamma<y_{-n+k}$. In particular, if all interpolation points $y_{s}$ are positive $y_{s}>0$ then it is sufficient to take $\gamma=0$ (i.e. in this case the contour $\Gamma$ coincide with imaginary axis).

Similarly, in the case of the power function $F(z)=z^{p}$ (where the $p$ is an arbitrary real parameter with the restriction $-1<p<M+1$ ) we have biorthogonality property in the form

$$
\frac{\sin (\pi p)}{\pi} \int_{-\infty}^{0} \frac{(-x)^{p} U_{n}(x ; M, k) V_{m}(x ; M, k) d x}{W_{k M}(x)}=h_{n}(M, k), \delta_{n, m}
$$

We thus have

Theorem 6. The regular Padé interpolation scheme for the exponential and power functions on arbitrary interpolation grids $y_{s}$ (with above mentioned weak restrictions) leads to rational functions $U_{n}(z ; M, k), V_{m}(z ; M, k)$ which are biorthogonal with respect to the Fourier and Mellin integrals respectively.

Of course, compact explicit expressions for rational functions $U_{n}(z ; M, k), V_{n}(z ; M, k)$ can be found only for exclusive choice of the interpolation grid: arithmetic progression for the exponential function and geometric progression for the power function. Still, Theorem 6 allows to recognize all BRF with respect to the Fourier and Mellin integrals as arising in problem of the Padé interpolation for exponential and power function. Such integral realization may be useful in questions concerning asymptotic behavior of corresponding interpolants.

\section{Conclusion}

We showed that the simplest self-similarity condition for the Darboux transformations in GEVP generates 2 possible types of regular non-commutative algebras of dimension 2. Moreover, these two types correspond to biorthogonal rational functions which can be expressed in terms of (basic) hypergeometric functions. Under a simple restriction on the parameters of BRF we obtain solutions of the Padé interpolation problem for the exponential and power function. In these special cases the linear functional providing biorthogonality can be realized as Fourier and Mellin integrals.

It is useful to compare these results with similar ones obtained in theory of self-similar closure of the chain of Darboux transformations for the ordinary orthogonal polynomials 
[14], [15]. It is known that self-similar closure of the finite chain of the Darboux transformation in this case leads to so-called semi-classical orthogonal polynomial on uniform or exponential grids [15]. In the simplest case of closure after the first Darboux transformation we obtain Charlier or q-Charlier polynomials [11]. It is naturally to conclude that our constructed examples of BRF are "rational" analogues of the (q)-Charlier polynomials. It would be interesting to study further, more complicated possibilities of self-similar closure conditions for the Darboux transformations. One can expect the appearance of non-trivial algebras with non-linear commuting relations. Although many examples of explicit biorthogonal functions were constructed, still the general hierarchy of BRF is unclear. E.g., even in our simple examples of hypergeometric rational functions we were not able to find an integral representation for the corresponding linear functional in the general case. The relation of BRF with the theory of the Padé interpolation is also a very fruitful area of investigation. Connection between the usual Padé approximation and orthogonal polynomials is well known (see, e.g. [3]) and goes back to classical works by Chebyshev and Stieltjes. For the Padé interpolation, however, we are only at the starting point of a similar theory. Some first results can be found in [17], [23].

\section{References}

[1] Artin M and Schelter W F, Graded algebras of global dimension 3, Advances in Math. 66 (1987), 171-216.

[2] Atkinson F V, Multiparameter Eigenvalue Problems, Academic Press, 1972.

[3] Baker G A and Graves-Morris P, Padé approximants. Parts I and II., Encyclopedia of Mathematics and its Applications, 13, 14. Addison-Wesley Publishing Co., Reading, Mass., 1981.

[4] Chihara T S, An introduction to orthogonal polynomials, Mathematics and its Applications, Vol. 13, Gordon and Breach Science Publishers, New York-London-Paris, 1978.

[5] Erdélyi A, Magnus W, Oberhettinger F and Tricomi F, Higher transcendental functions. Vols. I, II, Based, in part, on notes left by Harry Bateman, McGraw-Hill Book Company, Inc., New York-Toronto-London, 1953

[6] Gantmaher F R, The Theory of Matrices, vols. 1-2, Chelsea, NY, 1959.

[7] Gasper G and Rahman M, Basic Hypergeometric Series, Cambridge University Press, Cambridge, 1990.

[8] Irving R S, Prime ideals of Ore extensions over commutative rings, II, J. Algebra $\mathbf{5 8}$ (1979), 399-423.

[9] Iserles A, Rational interpolation to $\exp (-x)$ with application to certain stiff systems. SIAM J. Numer. Anal. 18 (1981), 1-12.

[10] Ismail M and Masson D, Generalized orthogonality and continued fractions, J. Approx. Theory 83 (1995), 1-40.

[11] Koekoek R and Swarttouw R, The Askey-scheme of hypergeometric orthogonal polynomials and its q-analogue, Delft University of Technology, Faculty of Information Technology and Systems, Department of Technical Mathematics and Informatics, Report no. 98-17, 1998. 
[12] Meinguet J, On the solubility of the Cauchy interpolation problem, Approximation Theory (Proc. Sympos., Lancaster, 1969), Academic Press, London, 1970, 137-163 .

[13] Rieffel M, Quantum Deformations for Actions of $R^{d}$, Mem. Amer. Math. Soc. 506, American Mathematical Society, Providence, 1993.

[14] Spiridonov V and Zhedanov A, Discrete-time Volterra chain and classical orthogonal polynomials, J. Phys. A 30 (1997), 8727-8737.

[15] Spiridonov V, Vinet L and Zhedanov A, Spectral transformations, self-similar reductions and orthogonal polynomials, J. Phys. A 30 (1997), 7621-7637.

[16] Spiridonov V and Zhedanov A, Generalized eigenvalue problem and a new family of rational functions biorthogonal on elliptic grids, Special functions 2000, Kluwer, 2001, $365-388$.

[17] Spiridonov V and Zhedanov A, To the theory of biorthogonal rational functions, RIMS Proceedings, 2003.

[18] Wilkinson J H, The Algebraic Eigenvalue Problem, Clarendon Press, Oxford, 1965.

[19] Zhedanov A S, On the realization of the Weyl commutation relation $H R=q R H$, Phys. Lett. A 176 (1993), 300-302.

[20] Zhedanov A, Biorthogonal rational functions and the generalized eigenvalue problem, $J$. Approx. Theory 101 (1999), 303-329.

[21] Zhedanov A, Biorthogonality of the Lagrange interpolants, J. Comput. Appl. Math. 172 (2004), 1-6.

[22] Zhedanov A, Explicit multipoint rational interpolation Padé table for exponential and power functions, CRM Proceedings and Lecture Notes 39 (2004), 285-298.

[23] A. Zhedanov, Padé interpolation table and biorthogonal rational functions, Proceedings of RIMS Workshop on Elliptic Integrable Systems, Kyoto, November 8-11, 2004, to be published. http://www.math.sci.kobe-u.ac.jp/HOME/noumi/EIS/zhedanov4.pdf 